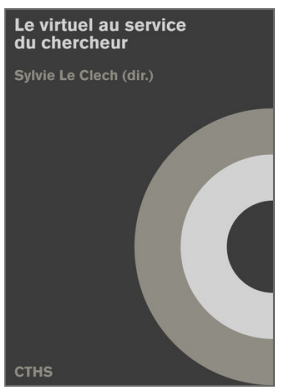

Sylvie Le Clech (dir.)

Le virtuel au service du chercheur

Éditions du Comité des travaux historiques et scientifiques

\title{
La reconstitution culinaire : une réalité virtuelle ou un rêve devenu réalité ?
}

\section{Sandrine Krikorian}

DOI : 10.4000/books.cths. 15338

Éditeur : Éditions du Comité des travaux historiques et scientifiques Lieu d'édition : Éditions du Comité des travaux historiques et scientifiques Année d'édition : 2021

Date de mise en ligne : 21 juin 2021

Collection : Actes des congrès nationaux des sociétés historiques et scientifiques EAN électronique : 9782735509300

\section{Q boooks}

http://books.openedition.org

\section{Référence électronique}

KRIKORIAN, Sandrine. La reconstitution culinaire : une réalité virtuelle ou un rêve devenu réalité ? In : Le virtuel au service du chercheur [en ligne]. Paris : Éditions du Comité des travaux historiques et scientifiques, 2021 (généré le 24 juin 2021). Disponible sur Internet : <http://books.openedition.org/ cths/15338>. ISBN : 9782735509300 . DOI : https://doi.org/10.4000/books.cths. 15338. 


\title{
La reconstitution culinaire : une réalité virtuelle ou un rêve devenu réalité ?
}

\author{
Sandrine Krikorian
}

1 Depuis quelques années, le goût du grand public pour la cuisine et la pâtisserie est de plus en plus marqué et semble inexorable. Les nombreuses émissions télévisées de cuisine montrent à quel point le grand public y est sensible. Cependant, l'existence d'émissions culinaires cathodiques n'est pas récente, mais date du début de la création de la télévision française. Il en est ainsi de la première émission, Les recettes de monsieur X, réalisée en 1953 par Jean d'Arcy - bien qu'elle ne connût pas un franc succès. Par contre, la popularité fut au rendez-vous pour Art et magie de la cuisine, réalisée en 1954 avec Raymond Oliver (chef du restaurant Le Grand Véfour à Paris) et Catherine Langeais (speakerine), qui diffusait un certain modèle culinaire urbain, principalement bourgeois, auprès des ménagères. Les émissions culinaires se sont de plus en plus développées et diversifiées. Dans les années 1970 avec La Grande Cocotte, des chefs réputés comme Paul Bocuse se prêtent au jeu, révélant certaines de leurs recettes au grand public. Dans les deux dernières décennies du $\mathrm{xx}^{\mathrm{e}}$ siècle, l'une des émissions les plus marquantes est La Cuisine des mousquetaires avec Maïté (Marie-Thérèse Ordonez) et sa comparse Micheline Banzet: à l'origine simple émission régionale sur France 3 Aquitaine, elle passe ensuite sur la chaîne nationale France 3 et doit son succès à la personnalité de Maïté, incarnant l'authenticité de la cuisine du terroir, et à son décalage avec les standards culinaires de l'époque. Par ailleurs, la personnalité de JeanPierre Coffe marque les écrans et les esprits par son côté haut en couleur et ses avis tranchés quant à la question du bien-manger. Une émission phare des années 2000 est Bon appétit, bien sûr! avec le chef Joël Robuchon qui dévoile chaque jour une recette, accompagné par un confrère. Au cours des années 2010, des émissions comme Top Chef, Master Chef ou, dans un style quelque peu différent, Le Meilleur Pâtissier, renouvellent le genre, mêlant les concepts de l'émission culinaire, du radio-crochet et de la téléréalité ${ }^{1}$. 
2 Si la reconstitution historique est éphémère, la reconstitution culinaire l'est encore davantage, car cuisine, gastronomie et arts de la table le sont par définition ${ }^{2}$. La reconstitution culinaire, dans son aspect purement gustatif, permet de mettre à la portée de tout un chacun ce qui était jadis réservé à une élite. On assiste à une démocratisation de la gastronomie "des riches». Si tous les mets ne sont pas facilement réalisables, certaines recettes sont plus abordables et plus aisément faisables que d'autres.

3 Dans cette optique, plusieurs questions se posent. Qu'entend-on exactement par le terme de "reconstitution culinaire»? S'agit-il d'une réalité virtuelle ou d'un rêve devenu réalité ? Quelle est la place des chercheurs au sein de cette discipline?

4 D'un point de vue purement scientifique, son champ disciplinaire est on ne peut plus restreint, puisqu'à l'échelle de l'histoire, son existence et les études qui en découlent sont récentes bien qu'en perpétuelle évolution. Cet article est le fruit du croisement d'une démarche à la fois théorique - fondée sur les méthodologies scientifiques issues des sciences humaines et sociales - et expérimentale, prenant appui sur l'interrelation de plusieurs années d'étude à partir d'observations, de discussions, de questionnements et d'échanges plus ou moins formels avec les acteurs concernés - professionnels du tourisme, associations, groupes folkloriques, etc. -, et tenant compte de la démarche et des spécificités qui leur sont propres.

5 Aussi, cet article se développe selon deux axes: le premier, théorique, partant de données lexicographiques, historiques, psychologiques et sociologiques, le second ayant pour base des considérations plus empiriques et expérimentales, dans le but de comprendre à la fois les aspects les plus abstraits et les plus concrets de ces éléments et de faire le lien entre eux.

\section{Démarche théorique : définitions, terminologies et théories scientifiques}

6 La discipline tient à la fois du virtuel et du réel. Selon le Centre national de ressources textuelles et lexicales (CNRTL) ${ }^{3}$, les sens philosophiques, qui nous concernent ici, indiquent pour le mot virtuel : « Qui possède, contient toutes les conditions essentielles à son actualisation "; et pour le mot réel : "Qui existe d'une manière autonome, qui n'est pas un produit de la pensée. Qui est dégagé de la subjectivité du sujet. » Plus loin dans la définition est indiqué : "Qui existe, qui se produit effectivement, qui n'est pas un produit de l'imagination. Qui appartient à la nature, qui a lieu en tant que processus physique. » La réalité, en art culinaire comme ailleurs, est avant tout une question d'ordre philosophique - Qu'est-ce que la réalité ? - et empreinte de subjectivité. Si l'on prend l'exemple de la peinture, on parle de peintres de la réalité à propos d'artistes figurant des personnes du peuple ${ }^{4}$. La réalité du peuple n'est pas la même que celle des élites. De même, la réalité est différente pour tout un chacun.

7 Concernant la reconstitution culinaire, le CNRTL ne propose pas de définition de cette expression, mais parle toutefois de reconstitution historique. Si la « reconstitution » est définie comme l'« action de reconstituer, de former de nouveau ce qui est disparu », la définition de la reconstitution historique est la suivante :

LITT., SPECTACLES. Reconstitution (historique). Évocation du passé ou d'un événement appartenant à l'Histoire que l'on fait revivre par une composition 
humaine, artistique. «Nous n'avons pas besoin pour nous imaginer ce qu'était, vivante et dans le plein exercice de ses fonctions sublimes, une cathédrale du XIII ${ }^{\mathrm{e}}$ siècle, d'en faire comme du théâtre d'Orange, le cadre de reconstitutions, de rétrospectives exactes peut-être, mais glacées » (Proust, Chron., 1922, p. 153).

La catégorisation de la reconstitution historique est totalement inscrite dans le domaine du spectacle. Nous sortons donc de ce fait du cadre strictement scientifique et philosophique et entrons dans un domaine artistique. Il s'agit là d'un aspect primordial à prendre en considération : comment et de quelle façon la reconstitution historique de façon générale, et la reconstitution culinaire en particulier, peuvent-elle être traitées avec une seule et unique rigueur scientifique alors qu'elles font partie du domaine artistique obéissant à ses propres codes et règles? Plus simplement, comment mettre en valeur esthétiquement un fait sans en altérer la réalité historique ? C'est bien là tout le nœud du problème.

9 L'acception première du terme «culinaire », logiquement et étymologiquement, découle du mot de cuisine et le mot " gastronomique » lui est peu ou prou synonyme dans les faits. Toutefois, réduire la reconstitution culinaire au simple aspect gustatif démontrerait une méconnaissance de la portée à la fois psychologique, sociale et culturelle de l'action de manger. Certes, la cuisine en tant que telle est bien évidemment un besoin primaire, situé au bas de la pyramide de Maslow et nécessaire à toute vie ${ }^{5}$. Ceci est également à mettre en lien avec une transmission intergénérationnelle, souvent orale, dans le but de perpétuer une tradition, un rite, réappropriés à l'identique. Pierre Benghozi parle d'un «patrimoine psychique familial [qui] est reçu par une génération, mémorisé, historicisé, transformé, élaboré et transmis à la nouvelle génération ${ }^{6} »^{7}$.

Pierre-Jean-Baptiste Legrand d'Aussy (1737-1800), dans son Histoire de la vie privée des Français, l'explique parfaitement :

«L'histoire de la vie privée d'un Peuple doit, comme celle de l'homme, commencer par le premier \& le plus pressant de ses besoins. La nécessité d'un logement ou d'un habit n'est que secondaire. Il est des tems où la Nature nous dispenserait de l'un \& de l'autre ; mais il n'est aucun jour où elle nous dispense de nourriture ; \& c'est sous peine de la faim \& de la mort qu'elle ordonne à tout être vivant de s'en procurer une $^{8}$.»

11 Les historiens et autres chercheurs en sciences humaines et sociales spécialisés dans l'alimentation, la cuisine, la gastronomie, les arts de la table, ont cependant démontré depuis longtemps déjà la dimension intellectuelle et mentale, consciente et inconsciente, de la cuisine. D'ailleurs, si elle est une discipline récente, un récit plus ancien témoigne de cet intérêt intergénérationnel pour cette pratique. C'est le cas de la relation faite dans ses Souvenirs par Élisabeth Vigée-Lebrun qui, en 1788, à la suite de la lecture de l'ouvrage fictif de l'abbé Barthélémy sur le Voyage du jeune Anarchasis en Grèce publié la même année, organise un "souper grec » qui a défrayé la chronique et dont voici un extrait de la narration ${ }^{9}$ :

«Je fis aussitôt monter ma cuisinière, je la mis bien au fait, et nous convînmes qu'elle ferait une certaine sauce pour la poularde, et une autre pour l'anguille. Comme j'attendais de fort jolies femmes, j'imaginai de nous costumer à la grecque, afin de faire une surprise à Monsieur de Vandreuil et Madame Boutin, que je savais ne devoir arriver qu'à dix heures. Mon atelier, plein de tout ce qui me servait à draper mes modèles, devait me fournir assez de vêtements, et le Comte de Parois, qui logeait dans ma maison, rue de Cléry, vint précisément chez moi, ce jour-là, vers quatre heures. Je lui fis part de mon projet, en sorte qu'il m'apporte une quantité de 
coupes, de vases, parmi lesquels je choisis. [...] Une lampe suspendue donnait une forte lumière sur la table : enfin, tout était préparé, jusqu'à mes costumes, lorsque la fille de Joseph Vernet, la charmante Madame Chalgrin, arriva la première. Aussitôt, je la coiffe, je l'habille. Puis vint Madame Vigée, ma belle-sœur, qui, sans être aussi jolie, avait les plus beaux yeux du monde, et les voilà toutefois métamorphosées en véritables Athéniennes. Le Brun-Pindare entre : on lui ôte sa poudre, on défait ses boucles de côté, et je lui ajuste sur la tête une couronne de laurier. Puis vint le Marquis de Cubières. Tandis que l'on va chercher chez lui une guitare qu'il avait fait monter en lyre dorée, je le costume: je costume aussi M. de Rivière Guiguené et Caudet, le fameux sculpteur [...].

Outre les deux plats dont je vous ai déjà parlé, nous avions pour souper un gâteau fait avec du miel et du raisin de Corinthe et deux plats de légumes. À la vérité, nous bûmes ce soir-là une bouteille de vieux vin de Chypre dont on m'avait fait présent ; mais voilà tout pour l'excès. Nous n'en restâmes pas moins à table, où Le Brun nous récita plusieurs odes d'Anacréon qu'il avait traduites, et je ne crois pas avoir jamais passé une soirée aussi amusante. »

Cette citation montre à quel point la reconstitution culinaire envisagée et réalisée en cette fin du XVIII ${ }^{e}$ siècle dépasse largement le cadre de la simple expérience gustative et englobe une dimension mentale primordiale et indéniable. Aussi, le terme de reconstitution culinaire ne peut être restreint à sa seule signification alimentaire et doit être employé dans son acception la plus large, englobant les facteurs culturels, historiques et mentaux cités supra.

\section{Démarche expérimentale, reconstitution culinaire et grand public : l'exemple provençal}

13 La reconstitution culinaire est protéiforme: recettes d'antan, dioramas et reconstitutions muséographiques, ou encore intégration dans des manifestations folkloriques et des spectacles. L'intérêt de la reconstitution culinaire est qu'elle peut être un support privilégié de diffusion de l'information, un terrain d'entente où savoir scientifique et grand public se rencontrent, par exemple dans les musées. Elle favorise la transmission de cette connaissance universitaire à tous. Dans une volonté de cohérence, l'aire géographique déterminée concerne pour cette étude le seul département des Bouches-du-Rhône.

\section{Les tables reconstituées des musées}

14 En Provence, le Museon Arlaten, depuis sa création et ce jusqu'à sa fermeture pour travaux, proposait un diorama reconstituant une veillée calendale avec une table chargée des mets consommés par les Provençaux lors du gros souper. Le Musée provençal - anciennement le musée du Terroir marseillais - situé à Château-Gombert, présente également la table d'un Noël en Provence au moment des treize desserts. La volonté est purement ethnographique et la fonction essentiellement didactique ${ }^{10}$.

Le diorama du Museon Arlaten montre la cérémonie du cacho fiò (bénédiction de la bûche) - rite se déroulant la veille de Noël avant le gros souper - et la table sur laquelle sont disposés nappes, blé planté le 4 décembre, jour de la sainte Barbe, et plats: escargots, cardes, céleri, muge aux olives, fougasse, confiture. 

montre le deuxième service du repas : sur des nappes de grandes dimensions et sur un meuble attenant se trouve le blé de la sainte Barbe au milieu des nougats noir et blanc, pompe à l'huile, amandes, noisettes, noix, raisins frais, etc. reconstitution historique et culinaire, il faut toujours garder à l'esprit que cette dernière, même dans un musée, revêt un aspect interprétatif dû à la personne qui met en scène la table, tout comme un journaliste choisirait son angle d'attaque dans l'écriture d'un article.

dans le cas du Museon Arlaten, Frédéric Mistral, fondateur du Félibrige et créateur du musée, avait pour but de mettre en avant le patrimoine régional, même de créer une identité régionale. Et encore, comme dans la langue - puisqu'il se fonde sur le provençal rhodanien - ou le costume d'Arles sur lequel la mode et les mentalités de Paris ont une grande influence, un choix et une codification ont été opérés et transparaissent à travers le diorama du musée arlésien. Quant au musée de ChâteauGombert présentant les treize desserts - dont le nombre n'avait d'ailleurs pas été indiqué à l'origine par Frédéric Mistral -, il s'agit là encore d'un musée visant à mettre en valeur le terroir et les traditions. C'est donc cette approche qui a été privilégiée, ce qui n'est pas le cas de tous les musées.

eut ainsi prendre un autre exemple, exclusivement marseillais, et particulièrement éclairant. Les reconstitutions des tables qui se trouvaient, l'une au musée de la Faïence lorsque celui-ci était au château Pastré, et l'autre actuellement présentée au château Borély depuis son déménagement, lorsque Marseille est devenue capitale européenne de la culture en 2013. Dans les deux cas, il s'agit de la reconstitution d'une table du $\mathrm{XVIII}^{\mathrm{e}}$ siècle avec des services quasiment identiques, mais dont la scénographie et certains éléments diffèrent.

La table reconstituée dans l'ancien musée de la Faïence mettait surtout l'accent sur l'aspect purement esthétique avec la nappe et la décoration: le surtout de table était agrémenté d'un ensemble floral et des festons de fleurs étaient accrochés à la nappe ${ }^{11}$. D'autres petites pyramides florales étaient disposées de part et d'autre de la table. Assiettes, plats et pots à oilles complétaient cet arrangement disposé, logiquement et avec symétrie de part et d'autre du surtout ${ }^{12}$.

21 La reconstitution de la table dans la salle à manger du musée Borély, à laquelle j'ai participé en tant que conseillère scientifique, tend vers la plus grande exactitude possible concernant l'ordonnancement général - place des couverts, etc. On peut néanmoins regretter le choix muséographique d'une table rétroéclairée plutôt qu'un recours à une véritable nappe, qui aurait permis de mettre en avant la fonction du linge de table et des fleurs dans la décoration. Le but est de reconstituer le plus précisément possible la présentation du premier service d'un repas au XVIII ${ }^{\mathrm{e}}$ siècle. Au premier plan se trouvent quatre assiettes accompagnées de couverts - couteaux, fourchettes, cuillères. D'autres assiettes ont été disposées sur le pourtour de la table. Le centre de celle-ci est décoré d'un surtout de la manufacture Joseph Fauchier datant du XVIII ${ }^{\mathrm{e}}$ siècle, de part et d'autre duquel sont disposés de façon symétrique pots à oilles et plats oblongs ${ }^{13}$ (fig. 1). 
Fig. 1. - Reconstitution d'une table du XVIII e siècle, actuel musée de la Faïence, château Borély (Marseille, Bouches-du-Rhône).

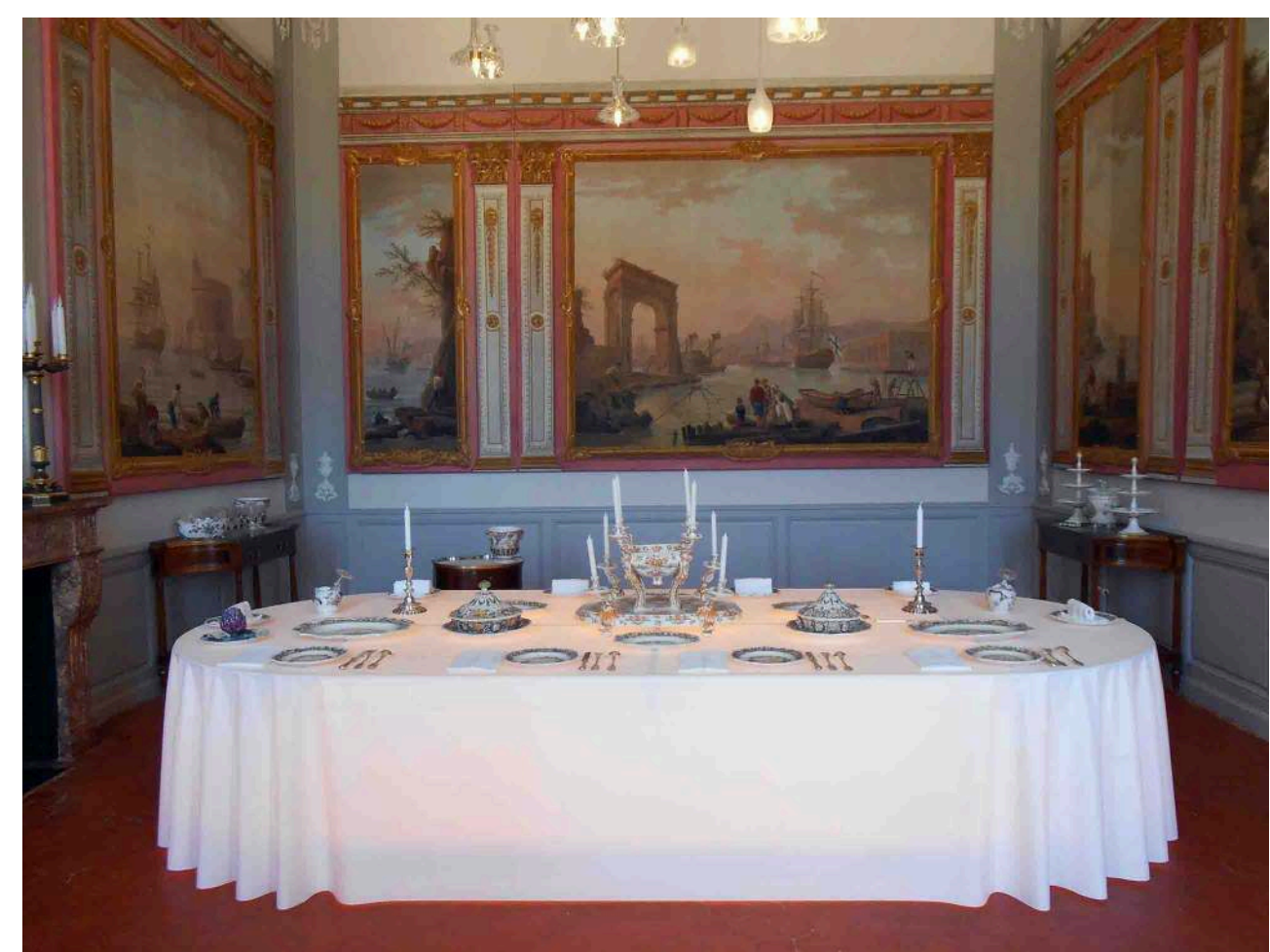

(c) S. Krikorian.

La comparaison entre ces deux tables montre que l'effet visuel, ainsi que les informations apportées, sont différents. La reconstitution est soumise non seulement à des fondamentaux scientifiques, mais aussi à un parti pris scénographique, pédagogique et didactique, en fonction non seulement du matériel de base à la disposition de l'expert, mais aussi des éléments qu'il préfère valoriser.

On le constate donc: même la plus précise des reconstitutions possède un aspect quelque peu personnel - sans aller jusqu'à le qualifier de subjectif - dans les choix de cet expert. À cela s'ajoute le "thème » du musée dans lequel la reconstitution est réalisée : celui des traditions populaires dans le cas du Museon Arlaten et du musée du Terroir marseillais de Château-Gombert, ou celui des arts de la table au XVIII siècle, dans le cas du musée de la Faïence situé aujourd'hui dans le château Borély.

Pour résumer, lorsque l'on analyse la reconstitution d'une scène et/ou d'une table dans un musée, il faut prendre en compte les contraintes purement matérielles, le lieu où se fait la reconstitution, le type de musée dont il s'agit ou encore le choix didactique, et esthétique, de l'expert qui préside à cette reconstitution.

D'autres types de reconstitutions culinaires, qui ne sont généralement pas le fait du spécialiste, engagent donc d'autres problématiques: les reconstitutions faites par des amateurs lors de fêtes.

\section{Manifestations et fêtes}

26 Aujourd'hui dans les Bouches-du-Rhône, certaines manifestations festives, tenant davantage du folklore que de la reconstitution historique exacte et rigoureuse, 
intègrent l'aspect gastronomique dans leurs fêtes. De l'Antiquité à la Renaissance, avec cependant une prédilection pour la période médiévale, ces fêtes se déroulent aux périodes printanières et estivales et font partie du paysage touristique provençal depuis plusieurs années.

Malheureusement, la problématique à laquelle nous sommes confrontés de nos jours est que, parfois, la démocratisation de ces reconstitutions culinaires dans les fêtes est le fait d'amateurs, certes passionnés, mais non de chercheurs expérimentés spécialistes du sujet. Conséquemment, malgré leur meilleure volonté et la bonne foi de leur implication dans les recherches entreprises et les informations transmises, la démarche scientifique n'est bien évidemment pas celle d'un chercheur professionnel. D'ailleurs, il arrive souvent que les personnes reconstituant repas et banquets soient en demande d'informations les plus exactes possibles, afin de les transmettre au mieux. Certaines emploient d'ailleurs plus volontiers le terme d'« évocation » culinaire ou historique que celui de reconstitution. Néanmoins, il arrive que certaines erreurs, parfois grossières, soient commises lors de la transmission du message - comme j'en ai malheureusement souvent eu la preuve en écoutant les discours lors de ces fêtes. La plus importante d'entre elles concerne le cardinal de Richelieu qui aurait publié un édit en date du 13 mai 1610 promulguant l'utilisation du couteau à bout rond - information qu'on retrouve d'ailleurs répétée ad libitum sur internet -, ce qui est totalement faux à plus d'un titre. Rappelons simplement la chronologie: Richelieu a été le ministre de Louis XIII. Or Henri IV, père de Louis XIII, a été assassiné le 14 mai $1610 . .$. On le constate donc, dans ces conditions le risque d'une transmission erronée de la vérité scientifique est considérable.

Dès lors, quel parti prendre pour l'historien, l'anthropologue, le sociologue - en bref, le chercheur en sciences humaines et sociales - qui se trouve confronté à cette problématique? Peut-il intervenir ? Si oui, comment le faire?

Plusieurs facteurs sont à considérer. Il faut avant tout être conscient qu'il serait illusoire et vain, dans tous les sens du terme, de croire que la vérité historique est et sera toujours la préoccupation première de la majorité des acteurs et spectateurs de ces reconstitutions. En effet, la reconstitution culinaire apporte une part d'onirisme au quotidien; c'est une façon de voyager et de s'évader de la réalité de la vie, parfois difficile. Cet aspect est un besoin inhérent à tout être humain. La reconstitution culinaire et historique plaît au grand public tout simplement, car elle apporte du rêve. C'est là une des raisons de la place considérable qu'elle occupe dans les nombreuses manifestations à vocation festive.

30 De plus, parmi d'autres facteurs psychologiques et sociaux inhérents à ce genre de manifestations - outre les reconstitutions culinaires -, la volonté de rendre visible ce qui ne l'est plus et le désir de communion entre les participants sont primordiaux ${ }^{14}$.

31 Par conséquent, ces fêtes drainent un grand nombre de personnes dont la consommation à cette occasion entraîne des retombées pécuniaires non négligeables. Il est donc primordial de prendre ces données en compte. Nier ces aspects et ces besoins fondamentaux et prioritaires des protagonistes reviendrait à empêcher toute communication entre les chercheurs et le grand public. En effet, ce type de manifestations possède un aspect social indéniable. Aussi, au lieu de les percevoir comme des événements dénaturant la vérité historique, il serait peut-être préférable de les envisager comme un terrain d'entente, une façon de créer du lien entre les scientifiques et le grand public afin que celui-ci soit plus réceptif aux messages de 
réhabilitation historique que les chercheurs veulent faire passer. C'est du moins ce que mon expérience - en tant que chercheuse et enseignante, et professionnelle du tourisme et de la communication - m'a permis de constater, et ce depuis une vingtaine d'années.

Une interrogation se pose alors : comment les institutions scientifiques peuvent-elles se positionner et de quelle façon peuvent-elles intervenir pour que le message passe davantage auprès du grand public? La question mériterait d'être étudiée de façon approfondie par les membres des institutions en question et les spécialistes du sujet, afin de pouvoir apporter des solutions adaptées.

L'une des premières choses à considérer est la définition de l'expression de « reconstitution culinaire ». À travers les théories des sciences humaines et sociales et par l'argumentation d'exemples précis et concrets, il apparaît plus pertinent de parler non pas d'une "reconstitution culinaire ", mais de "reconstitutions culinaires». Plus qu'une simple pratique liée à ce que l'on mange, il s'agit en réalité d'un mélange entre les diverses qualités gustatives et la culture dans ses différentes acceptions - art de vivre, gastronomie, arts de la table, ambiance, rites sociaux...

De par son caractère protéiforme, la reconstitution culinaire est une réalité virtuelle. C'est le cas des reconstitutions visibles dans les musées qui mettent en valeur son patrimoine culinaire en fonction de leur destination - traditions ou objets d'art - et le choix didactique du concepteur de la reconstitution en fonction d'éléments matériels et pédagogiques.

Mais elle est aussi un rêve devenu réalité, principalement à travers les reconstitutions faites lors de manifestations et de fêtes grand public. En effet, étant réalisée par des amateurs passionnés et non par des scientifiques, l'importance de la réalité historique passe au second plan, au profit d'un divertissement social et convivial faisant voyager dans le temps, l'espace d'un instant, visiteurs et curieux.

C'est justement pour cette raison que dans les deux cas, la place du chercheur est prédominante et ne doit pas être réduite dans la transmission du savoir, contrairement à ce qui est hélas trop souvent le cas de nos jours. Même si l'on doit faire preuve de circonspection et de discernement, la reconstitution culinaire peut permettre la transition entre le passé, l'histoire et l'avenir, ainsi qu'entre le réel et le virtuel.

Le virtuel peut également être envisagé sous une autre forme. En effet, avec l'utilisation grandissante des nouvelles technologies, des expérimentations et conceptions inédites sont à envisager telles que la réalité virtuelle. Malgré le lot de difficultés et de problématiques qui en découleront inévitablement, le recours à ces dernières peut offrir d'autres possibilités de transmission de connaissances auprès du grand public. La place et le rôle de l'historien s'avéreront sans aucun doute plus nécessaires que jamais ${ }^{15}$. 


\section{BIBLIOGRAPHIE}

BENGHOZI Pierre, «Transmission généalogique de la trace et de l'empreinte : temps mythique en thérapie familiale psychanalytique ", Cahiers critiques de thérapie familiale et de pratiques de réseaux, vol. XXXVIII, $\mathrm{n}^{\circ}$ 1, 2007, p. 43-60. [URL : https://www.cairn.info/publications-de-PierreBenghozi--67383.htm] BERTRAND Régis et FOURNIER Laurent-Sébastien (dir.), Les fêtes en Provence autrefois et aujourd'hui, Aix-en-Provence, Presses universitaires de Provence, 2014.

CRIVELLO Maryline, « Du passé, faisons un spectacle ! Généalogies des reconstitutions historiques de Salon et Grans en Provence (XIX ${ }^{\mathrm{e}}-\mathrm{XX}{ }^{\mathrm{e}}$ siècles) », Sociétés \& Représentations, vol. XII, $\mathrm{n}^{\circ} 2,2001$, p. 225-234.

CRIVELLO Maryline, « Mémoire, médias, Méditerranée : la geste des temps. Les reconstitutions historiques, pratiques sociales et imaginaire du passé (France, $\mathrm{XVIII}^{\mathrm{e}}-\mathrm{XXI}{ }^{\mathrm{e}}$ siècles) », mémoire d'habilitation à diriger des recherches, Aix-en-Provence, 2008.

ENNÈs Pierre, «Le surtout de mariage en porcelaine de Sèvres du Dauphin (1769-1770) », Revue de l'art, $\mathrm{n}^{\circ} 76,1987$, p. 63-73.

HAROCHE-BOUZIGNAC Geneviève, Louise-Élisabeth Vigée-Le Brun : histoire d'un regard, Paris, Flammarion, 2011.

KRIKORIAN Sandrine, Bergers et moutons de la Crau à l'alpe : pastoralisme ovin et transhumance de la Préhistoire à nos jours, préf. Régis Bertrand, Paris, BoD, 2021.

KRIKORIAN Sandrine, «L'identité provençale est en pleine métamorphose », dans Fornia Ariane (dir), Provence : les sillons du soleil, Bruxelles, Éditions Nevicata, 2019, p. 79-89.

KRIKORIAN Sandrine, Tables des riches, tables du peuple : gastronomies et traditions culinaires en Provence du Moyen Âge à nos jours, Saint-Martin-de-Crau, Jean-Marie Desbois éditions-GénéProvence, 2014.

KRIKORIAN Sandrine, «Les surtouts de table au XVIII ${ }^{\mathrm{e}}$ siècle : un langage visuel artistique et patriotique », dans Corbier Mireille et Sauron Gilles (dir.), Langages et communication : écrits, images, sons, éd. numérique, Paris, Éditions du CTHS, « Actes des congrès nationaux des sociétés historiques et scientifiques ", 2017, p. 191-197.

LEGRAND D'AUSSY Pierre-Jean-Baptiste, Histoire de la vie privée des Français depuis l'origine de la Nation jusqu'à nos jours, Paris, Ph.-D. Pierres, 1782 (3 vol.).

MOSSER Monique, « Le souper grec de Mme Vigée-Le Brun », Dix-Huitième Siècle, n 15, 1983, p. $165-168$.

OLIVIER Roger, La cuisine en spectacle : les émissions de recettes à la télévision (1953-2012), INA, 2016.

\section{NOTES}

1. Pour une histoire des émissions culinaires télévisuelles, on peut se référer au livre d'O. Roger, La cuisine en spectacle [...].

2. Maryline Crivello, entre autres, dans «Mémoire, médias, Méditerranée [...] », pour son habilitation, étudie la question des reconstitutions historiques. Concernant plus 
spécifiquement la Provence, notons deux autres travaux : R. Bertrand et L.-S. Fournier (dir.), Les fêtes en Provence autrefois et aujourd'hui et M. Crivello, «Du passé, faisons un spectacle ! [...] », p. 225-234.

\section{3. http://www.cnrtl.fr/definition/}

4. Ainsi, en 1934, avait été organisée par Paul Jamot et Charles Sterling une exposition intitulée "Les peintres de la réalité au XVII ${ }^{\mathrm{e}}$ siècle ", révélant l'œuvre de Georges de La Tour. Une seconde exposition en 2006-2007 à l'Orangerie, "Les peintres de la réalité ", abordait le même sujet, sous la houlette du directeur du musée Pierre Georgel, replaçant la première exposition dans un contexte politique et intellectuel plus large et faisant découvrir les échos de ces œuvres chez les peintres ultérieurs, jusqu'au $\mathrm{xx}^{\mathrm{e}}$ siècle.

5. Cela montre l'implication des personnes, à la fois en tant qu'acteurs - au sein des associations par exemple -, mais aussi en tant que spectateurs de ces reconstitutions. Rappelons que la pyramide élaborée par le psychologue dans les années 1940 pose cinq besoins de base : physiologique (éléments physiques élémentaires tels que le fait de manger), de sécurité (stabilité de l'environnement), d'appartenance (statut social, interaction avec un groupe), d'estime (réussite, reconnaissance) et d'accomplissement de soi (développement personnel), auxquels a été ajouté lors de la décennie suivante, un sixième besoin: celui de transcendance. Le lien peut s'établir dans le besoin d'appartenance, sa dimension sociale, l'individu se définissant dans sa relation avec l'autre. Une identité commune se crée donc.

6. P. Benghozi, "Transmission généalogique de la trace et de l'empreinte [...]», p. 43-60.

7. Ce qui se passe à un niveau individuel se retrouve également à un niveau collectif. Nous entrons là dans la sphère de la psychologie sociale et dans la question de la dynamique de groupe, qui sont à rapprocher des théories psychanalytiques, notamment celle de l'inconscient collectif élaborée par Gustav Jung.

8. P.-J.-B. Legrand d'Aussy, Histoire de la vie privée des Français [...], t. I, p. 1

9. Pour une étude sur ce repas, voir l'article de M. Mosser, "Le souper grec de Mme Vigée-Le Brun ", p. 165-168. On peut également se référer à la monographie de G. Haroche-Bouzignac, Louise-Élisabeth Vigée-Le Brun [...], p. 164-166.

10. Pour plus de détails, voir S. Krikorian, Tables des riches, tables du peuple [...].

11. Le surtout de table est un élément disposé au centre de la table destiné à rester sur celle-ci tout au long du repas. Voir P. Ennès, "Le surtout de mariage en porcelaine [...]», p.63-73; voir aussi S. Krikorian, «Les surtouts de table au XVIII ${ }^{\mathrm{e}}$ siècle $[. .] »$, p. 191-197.

12. Le pot à oille est un accessoire de table contenant un ragoût dont l'étymologie vient de l'espagnol olla podrida, qui était un ragoût de la péninsule ibérique.

13. Pour une explication détaillée de l'ordonnancement de cette table, voir S. Krikorian, Tables des riches, tables du peuple [...].

14. S. Krikorian "L'identité provençale est en pleine métamorphose », p. 79-89 et Id., Bergers et moutons de la Crau à l'alpe [...].

15. Notre époque est marquée par une volonté de conserver les informations sur support numérique, même si aujourd'hui un problème se pose puisque les spécialistes s'interrogent justement sur la façon de conserver ce qui est mis sur les supports 
informatiques en raison de la vitesse avec laquelle les nouvelles technologies avancent. En effet, cette course effrénée au progrès technologique fait craindre une perte future de certaines informations, car les supports actuels sont, en définitive, les plus fragiles qu'a connus l'humanité si on les compare aux autres supports utilisés jadis: pierre, papier, parchemin, etc. Les tablettes antiques en pierre ont conservé les informations jusqu'à nous et même après nous. En sera-t-il de même avec les tablettes actuelles dans 5000 ou 6000 ans?

\section{RÉSUMÉS}

Les reconstitutions historiques, et plus spécifiquement la reconstitution culinaire, ont pris de l'ampleur depuis quelques années dans l'espace public - fêtes, télévision, cinéma. Quels sont les facteurs humains ayant permis et permettant un tel essor ? Quelles sont la part du réel et celle de l'imaginaire? Quels sont le rôle et la place du chercheur en sciences humaines et sociales historien, historien de l'art, sociologue, ethnologue, psychologue, etc. ? Quel parti pédagogique peut-on prendre ? Comment les reconstitutions culinaires peuvent-elles être davantage mises au service de ce lien entre réalité et fiction, entre spécialistes et grand public ? À travers le cas de la Provence, et plus particulièrement des Bouches-du-Rhône, ce sont autant de questions abordées ici de façon interdisciplinaire permettant de considérer la façon dont le réel et le virtuel s'entrecroisent et évoluent, ainsi que la place qu'occupe la reconstitution historique culinaire au sein de notre société actuelle.

\section{AUTEUR}

\section{SANDRINE KRIKORIAN}

Chercheuse indépendante 